\title{
Ixodes scapularis dystroglycan-like protein promotes Borrelia burgdorferi migration from the gut
}

\author{
Jeroen Coumou $^{1}$ (D) Sukanya Narasimhan ${ }^{2} \cdot$ Jos J. Trentelman $^{1} \cdot$ Alex Wagemakers ${ }^{1} \cdot$ \\ Joris Koetsveld ${ }^{1} \cdot$ Jasmin I. Ersoz $^{1} \cdot$ Anneke Oei $^{3} \cdot$ Erol Fikrig $^{2} \cdot$ Joppe W. Hovius $^{1}$
}

Received: 22 August 2015 /Revised: 9 October 2015 / Accepted: 10 November 2015 / Published online: 23 November 2015

(C) The Author(s) 2015. This article is published with open access at Springerlink.com

\begin{abstract}
The causative agent of Lyme borreliosis, Borrelia burgdorferi, is transmitted by Ixodes ticks. During tick feeding, $B$. burgdorferi migrates from the tick gut to the salivary glands from where transmission to the host occurs. $B$. burgdorferi-interacting tick proteins might serve as vaccine targets to thwart B. burgdorferi transmission. A previous screening for B. burgdorferi-interacting Ixodes scapularis gut proteins identified an I. scapularis putative dystroglycan protein (ISCW015049). Here, we describe the ISCW015049's protein structure and its cellular location in the tick gut in relation to $B$. burgdorferi migration. Secondly, in vivo $B$. burgdorferi-tick attachment murine models were performed to study the role of ISCW015049 during B. burgdorferi migration and transmission. In silico analysis confirmed that ISCW015049 is similar to dystroglycan and was named I. scapularis dystroglycan-like protein (ISDLP). Confocal microscopy of gut tissue showed that ISDLP is expressed on the surface of gut cells, is upregulated during tick feeding, and is
\end{abstract}

Electronic supplementary material The online version of this article (doi:10.1007/s00109-015-1365-0) contains supplementary material, which is available to authorized users.

Jeroen Coumou

j.coumou@amc.nl

1 Center for Experimental and Molecular Medicine, Academic Medical Center, University of Amsterdam, 1105

AZ Amsterdam, The Netherlands

2 Department of Internal Medicine, Yale University School of Medicine, 06511 New Haven, CT, USA

3 Department of Medical Microbiology, Academic Medical Center, University of Amsterdam, 1105 AZ Amsterdam, The Netherlands expressed significantly higher in infected ticks compared to uninfected ticks. Inhibition of ISDLP by RNA interference (RNAi) resulted in lower B. burgdorferi transmission to mice. In conclusion, we have identified a dystroglycan-like protein in I. scapularis gut that can bind to B. burgdorferi and promotes $B$. burgdorferi migration from the tick gut.

\section{Key messages}

- B. burgdorferi exploits tick proteins to orchestrate its transmission to the host.

- B. burgdorferi is able bind to an I. scapularis dystroglycanlike protein (ISDLP).

- Inhibition of ISDLP in ticks results in lower B. burgdorferi transmission to mice.

- ISDLP is a potential target to prevent Lyme borreliosis.

Keywords Tick-borne diseases $\cdot$ Lyme borreliosis $\cdot$ Ixodes scapularis · Borrelia burgdorferi · Pathogen transmission . Vaccination

\section{Introduction}

In the USA, Ixodes scapularis is the vector of Borrelia burgdorferi, the causative agent of Lyme borreliosis [1,2]. $B$. burgdorferi colonization of the tick gut can occur when uninfected Ixodes larvae acquire B. burgdorferi when feeding on a $B$. burgdorferi-infected animal [3]. B. burgdorferi anchors itself to the tick gut wall by expressing outer surface protein A (OspA), which binds to the tick receptor OspA (TROSPA) [4]. When a B. burgdorferi-infected I. scapularis nymph feeds on a vertebrate host, $B$. burgdorferi becomes metabolically active, changes its outer surface proteins, and migrates from the tick gut to the salivary glands [5]. Motility 
of $B$. burgdorferi appears not to be essential for exiting the gut, as described in a newly proposed model called "adherence-mediated migration" by Dunham-Ems et al. [6]. Dunham-Ems and colleagues observed that during tick feeding, B. burgdorferi spirochetes initially replicate in the lumen of the gut and remain non-motile. After approximately $24 \mathrm{~h}$, B. burgdorferi spirochetes transition into aggregates at the basal lamina of the gut. From here, a small percentage of $B$. burgdorferi penetrate the gut, followed by migration via the hemolymph to the salivary glands into the skin of the host [6].

The close interaction of $B$. burgdorferi with tick gut epithelial cells suggests potential interactions between the tick gut proteins and B. burgdorferi proteins that might be critical for B. burgdorferi growth in the tick gut and its egress from the gut - a critical step for successful transmission to the vertebrate host. B. burgdorferi-interacting tick gut proteins might thus be a vaccine targeted to prevent spirochete migration from the gut and preempt transmission. The advantage of using tick gut proteins as antitick vaccines is that migration of B. burgdorferi can be targeted early in tick feeding-even before spirochetes have been transmitted to the host. Of note, a vaccine against Lyme disease is currently not available for humans [7]. Since the last decade, tick salivary gland proteins and tick gut proteins have become a target of vaccine development as the tick plays a central role in B. burgdorferi transmission [8]. Immunization against salivary gland proteins introduced into the skin that facilitate tick feeding provided (partial) protection against B. burgdorferi transmission [9-11] as well as immunization against tick gut proteins $[4,12]$. One limitation of vaccine targeting tick gut proteins is that gut proteins might not provide an anamnestic response, since they are not presented to the host during a tick bite. Nonetheless, the future development of cocktail vaccines combining tick gut proteins that facilitate spirochete migration from the gut and salivary antigens that facilitate survival at the bite site might provide a robust impairment of $B$. burgdorferi transmission by simultaneously targeting spirochete egress from the gut and survival at the bite site. Recently, we used a yeast surface display (YSD) approach to screen for $B$. burgdorferi-interacting tick gut proteins and identified three putative $B$. burgdorferi-interacting gut proteins: ISCW008121, ISCW015049, and ISCW015135 [12]. One of them, ISCW008121, was shown to be a transmembrane fibronectin domain-containing I. scapularis protein that enabled B. burgdorferi adherence to the basal lamina of the gut to facilitate transmission [12]. In this study, we report the characterization of ISCW015049 and examine its vivo role in the context of B. burgdorferi transmission and assess its ability to serve as a transmission-thwarting vaccine.

\section{Methods}

\section{Animal experiments}

The rabbit immunized against recombinant $I$. scapularis dystroglycan-like protein (rISDLP) and mice used in the RNA interference (RNAi) experiments were housed and handled under the Guide for the Care and Use of Laboratory Animals of the National Institutes of Health. The animal experimental protocol was approved by the Yale University's Institutional Animal Care and Use Committee (protocol number 2008-07941, approval date: 31 March 2014). All animal infection experiments were performed in a biosafety level 2 animal facility, according to the regulations of Yale University. In addition, the mice that were used for immunization experiments were housed and handled under the approval of the Animal Care and Use Committee of the University of Amsterdam (DIX103179).

\section{Ticks}

I. scapularis nymphs and larvae were obtained from a tick colony at the Connecticut Agricultural Experiment Station in New Haven CT, USA, and ticks were maintained as described earlier [13]. Ticks were allowed to feed to repletion and RNA isolated from guts and salivary glands using TRIzol (Invitrogen, CA, USA) as described earlier [22]. Complementary DNA (cDNA) was synthesized using the iScript RT-PCR kit (Bio-Rad, CA, USA) and analyzed by quantitative PCR for the expression of tick actin and $B$. burgdorferi flab using gene-specific primers (Online Resource 1) and the iQ SYBR Green Supermix (Bio-Rad, Hercules, CA, USA).

\section{Identification of the full-length transcript of ISCW015049 and purification of rISDLP}

First-strand cDNA was synthesized from the total I. scapularis gut RNA using a 3'-RACE adapter. The RLM-RACE kit was used to identify the sequence at the $3^{\prime}$-end and 5 '-end according to the manufacturer's instructions (Invitrogen, CA, USA). The identification of the sequence at the 5 '-end was performed using ISDLP-specific primers (Online Resource 1). The fulllength sequence was assembled using the Web-based software SMART [10] (http://smart.embl-heidelberg.de). Purification of rISDLP was performed as described previously [8].

\section{ELISA assessment of rISDLP binding to $B$. burgdorferi membrane extract}

B. burgdorferi membrane extract purified as described previously [12] was coated $(1 \mu \mathrm{g} / \mathrm{ml})$ on high binding microtiter plates (Microlon, Greiner, Germany) overnight at room 
temperature (RT). Wells were blocked with PBS/1 \% bovine serum albumin (BSA) at RT for $1 \mathrm{~h}$ and incubated with rISDLP or rTSLPI (3-100 pmol/ml) diluted in PBS $/ 0.05 \%$ Tween 20/1 \% BSA for $1 \mathrm{~h}$. Wells were washed and incubated with 1:5000 diluted mouse anti-V5 HRP IgG. Bound antibody was detected using TMB as substrate (Thermo Scientific, IL, USA).

\section{Confocal microscopy}

Confocal microscopy to detect native ISDLP was performed as previously described [12]. Briefly, guts from nymphal ticks (B. burgdorferi-infected or uninfected) were dissected and fixed in $4 \%$ paraformaldehyde (PFA). Washed guts were incubated with rabbit anti-rISDLP antibody and bound antibodies detected using fluorescein isothiocyanate (FITC)-labeled affinity purified goat anti-rabbit IgG antibody (Sigma, MO, USA) and nuclei stained with propidium iodide or with TOPRO-3 iodide (Invitrogen, CA, USA). Control guts were incubated with IgG purified from rabbit anti-ovalbumin sera. Stained guts were visualized under a Zeiss LSM 510 Confocal microscope.

\section{Pixel intensity quantification}

Pixel intensities in the tetramethylrhodamine (TRITC) channel (as a measure of anti-rISDLP serum binding to tick gut ISDLP) or in the FITC channel of confocal images were quantified using the ImageJ $1.47 \mathrm{t}$ software. Confocal images of four individual guts were examined in each control and experimental group, and mean pixel intensities representing the average intensity of pixels in the region of interest were obtained in five different regions of each tick gut.

\section{Immunization of rabbits and mice against ISDLP}

Four- to six-week old New Zealand white rabbits were immunized subcutaneously with $30 \mu \mathrm{g}$ of rISDLP or ovalbumin in complete Freund's adjuvant (CFA) and boosted twice with $30 \mu \mathrm{g}$ of rISDLP or ovalbumin at weeks 3 and 6 in incomplete Freund's adjuvant (IFA). Test bleeds were obtained from ear veins 2 weeks after the final boost, and reactivity to recombinant rISDLP and ovalbumin was assessed by Western blot. Rabbits were euthanized, and serum was obtained by cardiac puncture. Polyclonal IgG was purified from the sera using the Melon Gel IgG purification kit (Thermo Scientific, IL, USA).

For immunization of mice, animals were immunized with $10 \mu \mathrm{g}$ of rISDLP or ovalbumin in CFA and boosted twice with $10 \mu \mathrm{g}$ of rISDLP or ovalbumin at weeks 2 and 4 in IFA. To address the role of rISDLP in B. burgdorferi transmission, eight $B$. burgdorferi $\mathrm{N} 40$-infected nymphs were placed on each immunized mouse. Nymphs were allowed to feed to repletion. Salivary glands and guts were dissected and combined in pools of two to three ticks for quantitative reverse transcription polymerase chain reaction (RT-PCR) as described earlier [9]. DNA was isolated from skin punch biopsies at 7, 14, and 21 days and from heart and joints 21 days post-tick detachment, and Borrelia burden was assessed by quantitative PCR as described [9].

\section{RNAi silencing of isdlp in B. burgdorferi-infected I. scapularis nymphs}

RNAi silencing of isdlp in ticks was performed as described before [9] using primers specific for isdlp with a T7 promoter sequence (Online Resource 1). Double-stranded (ds) isdlp double-stranded RNA (dsRNA) was synthesized using the MEGAscript RNAi kit (Ambion/Invitrogen, CA, USA). ds isdlp RNA or ds $g f p$ RNA ( $5 \mathrm{nl}, 3 \times 10^{12}$ molecules $/ \mathrm{ml}$ ) was injected into the anal pore of Borreliainfected nymphs as described earlier [9]. dsRNAinjected ticks were allowed to feed until repletion and weighed to assess feeding efficiency, and guts and salivary glands were dissected for messenger RNA (mRNA) isolation and quantitative RT-PCR as described above. B. burgdorferi burden in mice was assessed by quantitative PCR as described earlier [9].

\section{Statistical analysis}

The significance of the difference between the mean values of the groups was analyzed using a non-parametric two-tailed Mann-Whitney test or a two-tailed Student's $t$ test with the Prism 5.0 software (GraphPad Software, San Diego, CA, USA), and $p \leq 0.05$ was considered significant.

\section{Graphics}

Figures have been created using the Prism 5.0 software (GraphPad Software, San Diego, CA, USA) and Adobe Illustrator (San Jose, CA, USA).

\section{Results}

\section{Full-length ISCW015049 encodes a potential} transmembrane dystroglycan-like protein

The protein sequence present in the YSD screening yeast colony "Clone 3"-matched amino acids (aa) 1 to 201 of the protein annotated on VectorBase as "putative dystroglycan, ISCW015049." The complete sequence of ISCW015049 was confirmed from the I. scapularis gut extract using 3'-end and 5'-end RLM-RACE. Using mRNA from guts of fed I. scapularis nymphs, the start and stop codons of ISCW015049 were identified and the complete 
transcript of 2904 bp was sequenced (Fig. 1a). Identification of the full sequence revealed that the full length of ISCW015049 was 968 aa long, since one fragment of ISCW015049 that annotated as an intron (www. vectorbase.org) was actually found to be part of the ISCW015049 transcript. In silico analysis showed that fulllength ISCW015049 has a potential transmembrane domain and is similar to dystroglycan, a widely distributed protein involved in the linkage between the extracellular matrix and the cytoskeleton [14]. The protein sequence of fulllength ISCW015049 was 27.0, 28.7, and $26.7 \%$ identical to Drosophila melanogaster dystroglycan (NP_725523.3), Homo sapiens dystroglycan (AA81779.1), and Mus musculus dystroglycan (NP_001263423.1), respectively. Online programs for protein modeling (SMART) predicted that ISCW015049 has three dystroglycan-type cadherinlike (CADG) domains and two dystrophin-associated glycoprotein 1 (DAG1) domains (Fig. 1b). The 201 aa region identified in the YSD screen was predicted to be located on the extracellular region, which consists of one CADG domain and a C-terminal domain of $\alpha$ dystroglycan (Fig. 1b, c). Based on the similarity with dystroglycan, full-length ISCW015049 is henceforth referred to as the I. scapularis dystroglycan like protein (ISDLP) and has been submitted to GenBank (accession number KR782315).

\section{Production of recombinant ISDLP and confirmation of binding to $B$. burgdorferi}

We expressed the full-length protein transcript of ISDLP in a $D$. melanogaster expression system. Binding of purified recombinant ISDLP (rISDLP) (Fig. 1d) to B. burgdorferi was confirmed by an ELISA-based binding assay. We observed a dose-dependent increase of rISDLP binding to $B$. burgdorferi membrane extract compared to the control tick protein rTSLPI (Fig. 1e).

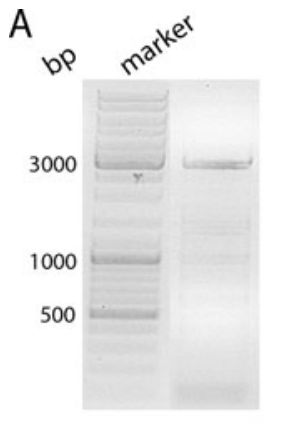

B Full length ISCW015049 (aa) ISCW015049 fragment present in YSD

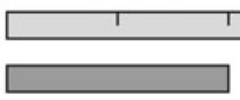

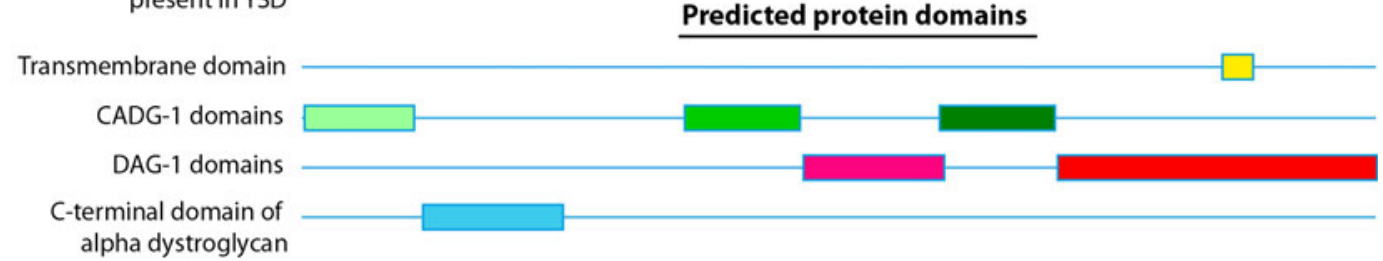
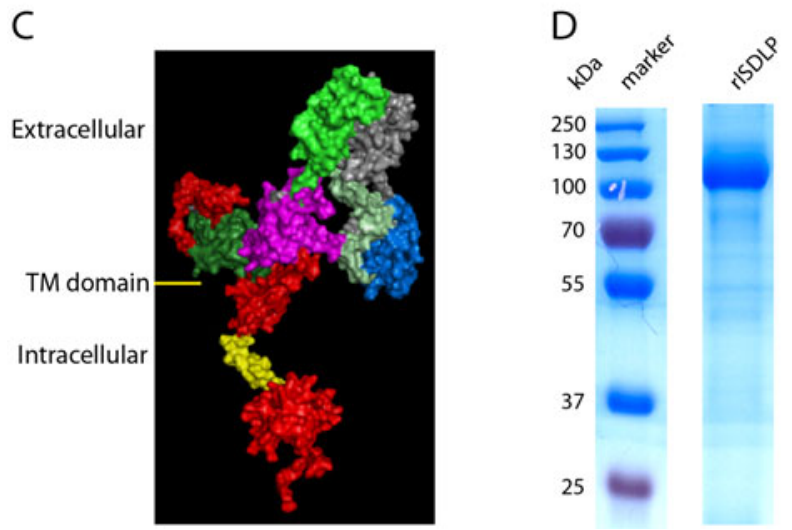

Fig. 1 Full-length sequence of ISDLP (GenBank accession number KR782315). a Guts from three fed I. scapularis were dissected, and RNA was extracted. Reverse transcription polymerase chain reaction (RT-PCR) was performed using primers based on the 3 '-end and $5^{\prime}$-end of ISCW015049 which were identified using the 3 '-end and 5'-end RLMRACE. The complete sequence was identified by sequencing of three contigs (1-604, 472-1575, and 1272-2904 bp), which were cloned into the PGEM-T Easy Vector. b Protein domains were predicted by SMART domain prediction (http://smart.embl-heidelberg.de) [25], TMHMM v2.0 software, and the conserved domain database of NCBI. If available, $E$ values are provided for each domain in parentheses. A potential transmembrane domain was found at aa $828-853$; three CADG-1

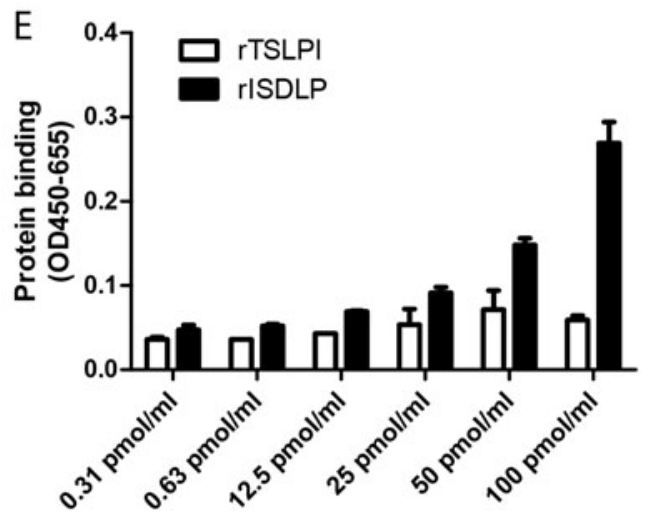

domains at aa 4-102 $\left(7.63^{-3}\right), 346-450\left(3.47^{-10}\right)$, and 576-680 $\left(2.01^{-6}\right)$ ; and two DAG-1 domains at aa 454-588 $\left(2.20^{-17}\right)$ and 683-968 (7.30 $\left.{ }^{70}\right)$; and a C-terminal domain of $\alpha$-dystroglycan that was identified at aa 111-236. c A 3D model of ISDLP was created using the Phyre2 software (http://www.sbg.bio.ic.ac.uk/phyre2) [26]. Six hundred seventy-six residues $(70 \%)$ were modeled at $>90 \%$ accuracy. d Purified Drosophila-expressed recombinant full-length ISDLP electrophoresed on SDS $10 \%$ polyacrylamide gel and stained with Coomassie blue. e ELISA assessment of dose-dependent binding of ISDLP to B. burgdorferi membrane protein extract-coated plates compared to rTSLPI, a tick protein that is known not to bind to $B$. burgdorferi 


\section{Localization of native ISDLP in the tick gut by confocal microscopy}

To study the expression and protein localization of native ISDLP, we generated antibodies against rISDLP by immunizing a rabbit with rISDLP. Unfed and partially fed (24 and $48 \mathrm{~h}$ ) $B$. burgdorferi and uninfected I. scapularis guts were collected, fixed with PFA, and probed with anti-ISDLP rabbit serum or anti-ova rabbit serum as a control. Binding of antibodies in the gut was visualized with immunofluorescence confocal microscopy. Comparing the binding of anti-rISDLP antibodies at the different time points by mean pixel intensity using the ImageJ software showed that ISDLP expression increases significantly during tick feeding (Fig. $2 \mathrm{a}-\mathrm{c}, \mathrm{e}$ ). Furthermore, increased binding was observed in B. burgdorferi-infected ticks compared to uninfected ticks. In line with our computer-based modeling of ISDLP (Fig. 1), Z-stack imaging suggested that ISDLP is represented both on the cell surface and in the cytosol (Fig. 2d).

\section{Immunization against rISDLP does not prevent B. burgdorferi transmission}

To test whether immunization against rISDLP would prevent B. burgdorferi transmission, we immunized eight mice against rISDLP and eight mice against ovalbumin. We achieved good IgG titer levels against rISDLP in the sera of mice that were immunized against rISDLP after immunization with complete Freund's adjuvants and two boosters with incomplete Freund's adjuvant (Fig. 3a). Two weeks after the second IFA boost, we placed eight ticks per mouse which were allowed to feed until repletion, ranging from 3 to 5 days. No difference was found in post-feeding tick weight compared to the ovalbumin group (Fig. 3b), nor did we observe a difference in $B$. burgdorferi migration to the salivary glands or in $B$. burgdorferi transmission to the host by RT-qPCR (Fig. 3c). Based on qPCR analysis, B. burgdorferi loads in skin tissue from the tick bite site (ears) as well as deeper tissue were similar between the rISDLP-immunized animals and the ovalbumin-immunized animals (Fig. 3d, e).

\section{isdlp RNAi reduces $B$. burgdorferi migration} to the salivary glands and transmission to the murine host

It is likely that in the active immunization experiment, the function of ISDLP in relation to B. burgdorferi migration and transmission remained unaffected by murine anti-ISDLP antibodies present in the gut during tick feeding. To circumvent the use of antibodies to provide an insight in the role of ISDLP during B. burgdorferi transmission, we performed another experiment in which isdlp expression in ticks was silenced by RNAi. We injected double-stranded (ds) isdlp RNA or ds $g f p$ RNA as a control in B. burgdorferi-infected
I. scapularis nymphs before placing four to five ticks on $\mathrm{C} 3 \mathrm{H} /$ $\mathrm{H} 3 \mathrm{~N}$ mice. The decrease of $i s d l p$ expression in the tick gut was confirmed by RT-qPCR (Fig. 4a). No difference in tick engorgement weights was observed between isdlp and the control ticks, indicating that reduced isdlp expression does not influence successful tick feeding (Fig. 4b). In contrast with the active immunization, significantly lower B. burgdorferi loads were detected in the salivary glands (Fig. 4c). Furthermore, qPCR on DNA from skin tissue showed significantly lower B. burgdorferi numbers in mice on which isdlp silenced ticks fed compared to the control group (Fig. 4d). However, B. burgdorferi loads were not significantly different at a later time point ( $t=14$ days) or in deeper tissues such as joint and heart, indicating that B. burgdorferi growth in the murine host is not affected by the reduced expression of $i s d l p$ in the tick (Fig. 4e).

\section{Discussion}

During tick feeding, while the tick gut adapts to cope with the uptake of blood, B. burgdorferi becomes metabolically active, replicates, and binds to hypertrophic and differentiating gut cells in order to cross the gut barrier $[6,15]$. The molecular mechanisms that direct the growth and migration from the tick gut and entry into salivary glands are only beginning to unfold [5]. B. burgdorferi has been shown to bind to host and tick proteins to facilitate its survival and dissemination [16]. To better understand vector $-B$. burgdorferi interactions, we performed a YSD screening to identify $I$. scapularis gut proteins that interact with B. burgdorferi. We identified four $B$. burgdorferi-interacting tick proteins of which one, Ixofin3D, has been previously described [12]. Here, we characterize one of the other three proteins, referred to as ISDLP, and assess its role in B. burgdorferi transmission.

Computer-based protein structure and function predictions showed that ISDLP is similar to the conserved transmembrane protein dystroglycan. Recombinant ISDLP binds to $B$. burgdorferi and is abundantly expressed on the surface of gut epithelial cells during tick feeding, which was in accordance with previous assessment of ISDLP expression by RTqPCR [12]. The function of ISDLP for I. scapularis has not yet been described. In other organisms, dystroglycan is part of the dystrophin-associated protein complex and is cleaved post-translationally into two subunits, $\alpha$ - and $\beta$-dystroglycan, that together form the dystroglycan complex [14]. The dystroglycan complex can bind to the extracellular matrix by binding to laminin. Furthermore, studies have shown that the dystroglycan complex is involved in cell adhesion-mediated signaling, tissue remodeling, and cell polarity and that $\beta$ dystroglycan is involved in MAPK signaling [17, 18]. The functional role of ISDLP on cell metabolism, cell signaling, 


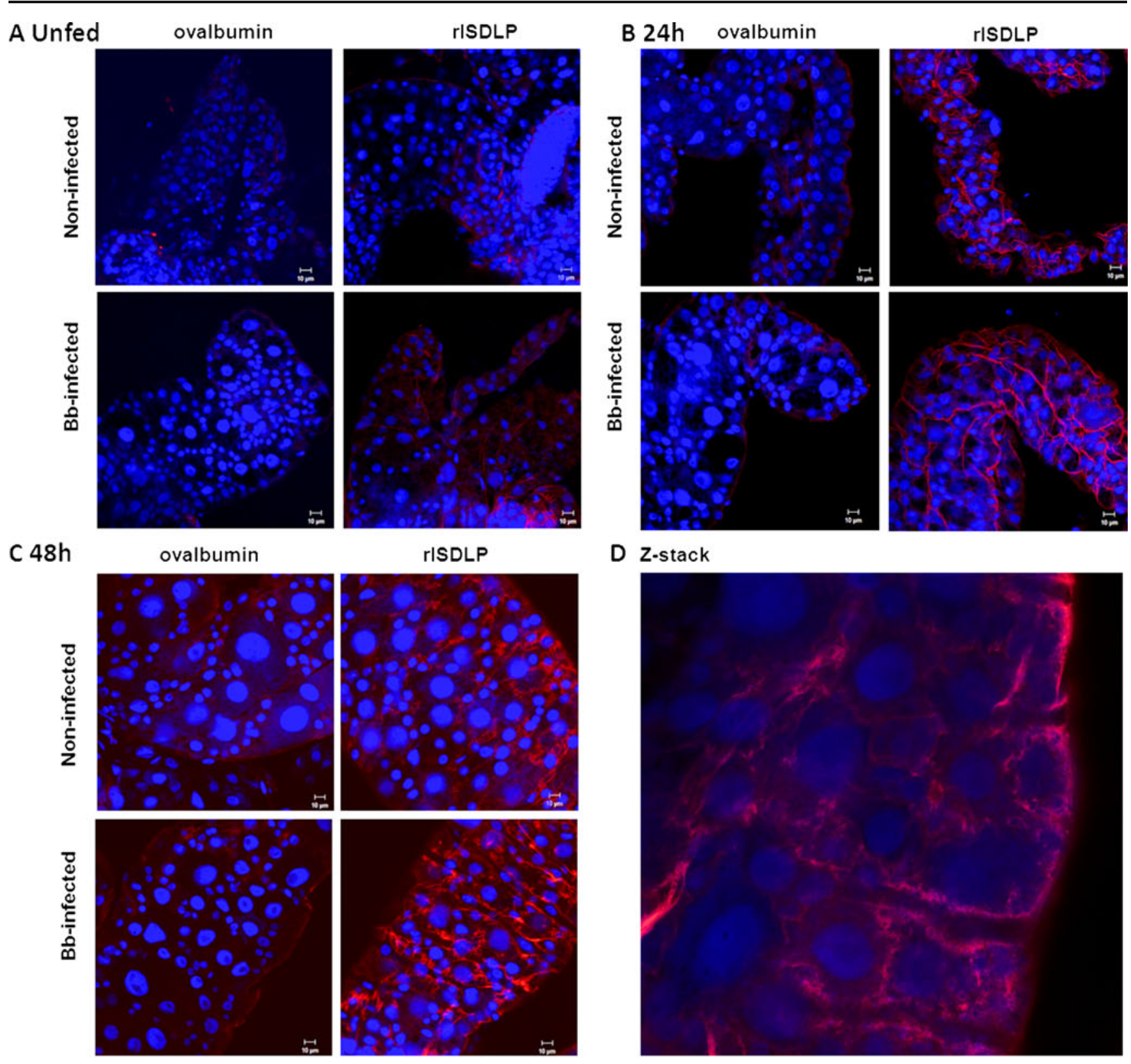

E

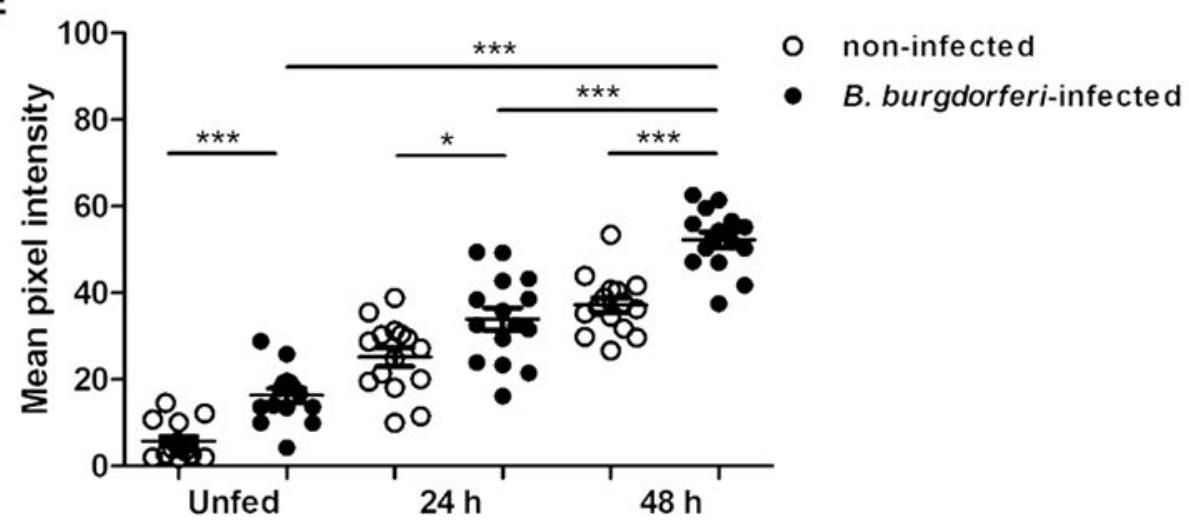


Fig. 2 ISDLP is a membrane-bound protein increasingly expressed in the gut during tick feeding. Gut nuclei and ISDLP were stained with TOPRO-3 (blue) and anti-rISDLP rabbit serum (TRITC, red), respectively. a-c Confocal microscopy of guts of unfed ( 24 and $48 \mathrm{~h}$ ) and fed uninfected and B. burgdorferi-infected I. scapularis nymphs. Magnification $\times 20$. Guts stained with anti-ovalbumin IgG (TRITC, red) served as antibody control. d A Z-stack (magnification $\times 63$ ) of a B. burgdorferi-infected gut after $48 \mathrm{~h}$ of feeding gut. e Mean pixel intensities of regions of interest in the TRITC channel (representing anti-rISDLP rabbit serum binding to ISDLP) of the confocal images obtained in $\mathbf{a}-\mathbf{c}$, as measured by the ImageJ software. Each data point represents one region of interest. The error bars represent mean $\pm \mathrm{SEM}$, and the mean values significantly different in a two-tailed non-parametric Mann-Whitney test are indicated by an asterisk $(p<0.05)$ or by three asterisks $(p<0.0001)$ or tissue remodeling during tick feeding remains to be defined.

RNAi-mediated decrease in the expression of ISDLP reduced $B$. burgdorferi transmission to the murine host. While B. burgdorferi load in the gut was not altered, B. burgdorferi load in the salivary glands was significantly reduced, suggesting that ISDLP might have a role in B. burgdorferi migration from the gut. There have been no previous reports on B. burgdorferi interactions with human dystroglycan, which is identified as a receptor for a number of viruses as well as for Mycobacterium leprae [19, 20]. B. burgdorferi is known to bind to extracellular matrix proteins such as decorin and fibronectin among others $[15,21]$, and we speculate that human dystroglycan could be a ligand for B. burgdorferi in humans
A
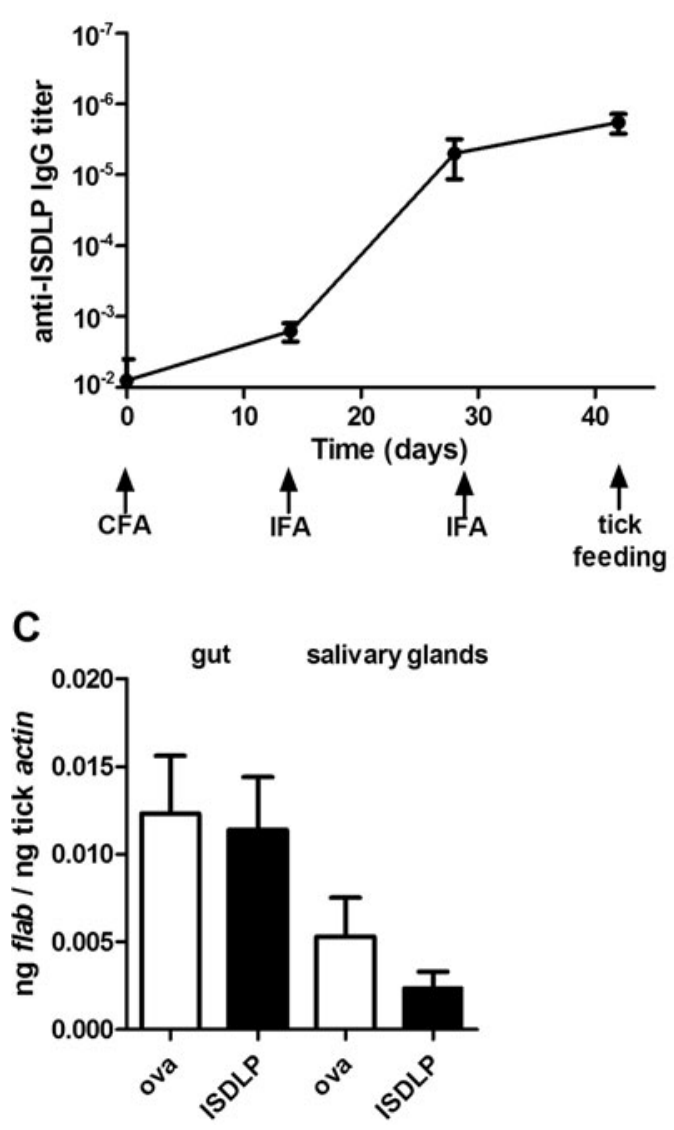

Fig. 3 Immunization against ISDLP does not have an effect on $B$. burgdorferi transmission to murine skin. Mice were actively immunized with rIxofin3D-PF or ovalbumin. Eight $B$. burgdorferi N40 ticks/mouse were placed and fed until repletion. Mice were sacrificed after 14 days of $B$. burgdorferi infection. a Mean IgG titer in the serum from animals vaccinated against rISDLP, diluted to $1: 10^{2}$ to $1: 10^{7}$ on ELISA-coated plates with rISDLP. The error bars represent mean \pm SEM. The cutoff for titer was calculated as an OD value of ova-

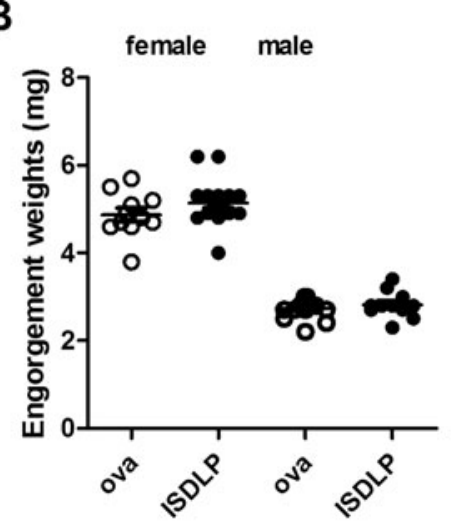

D

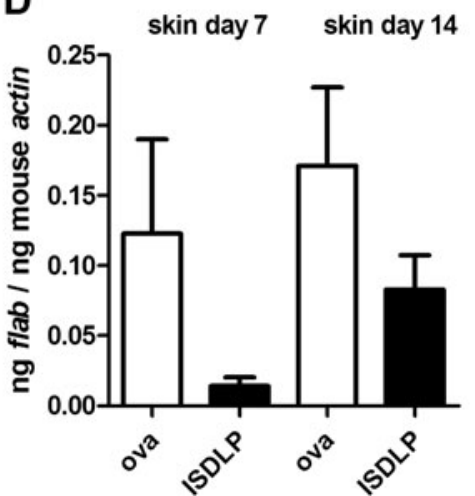

E

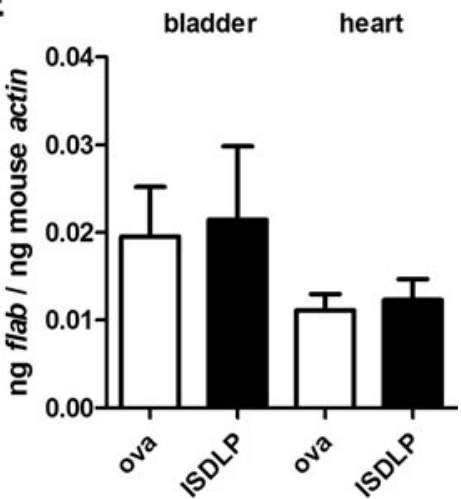

immune serum+3 SD. b Engorgement weights of ticks post-feeding. Each data point represents one tick. A tick was considered female when $>3.5 \mathrm{mg}$. c RT-qPCR assessment of $B$. burgdorferi burden in tick guts and salivary glands. d, e qPCR assessment of $B$. burgdorferi burden in the murine skin at 7 days and in the skin, bladder, and heart at 14 days posttick feeding. The experiment has been performed once with eight mice per group 


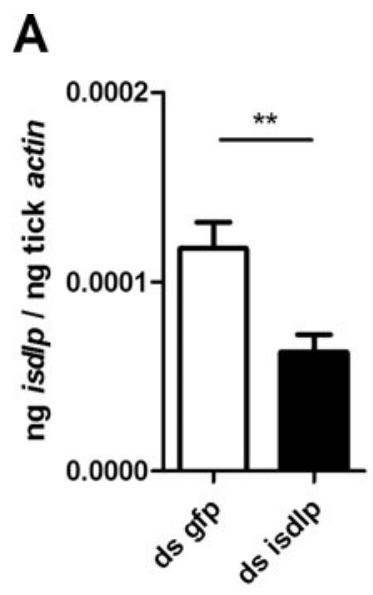

C

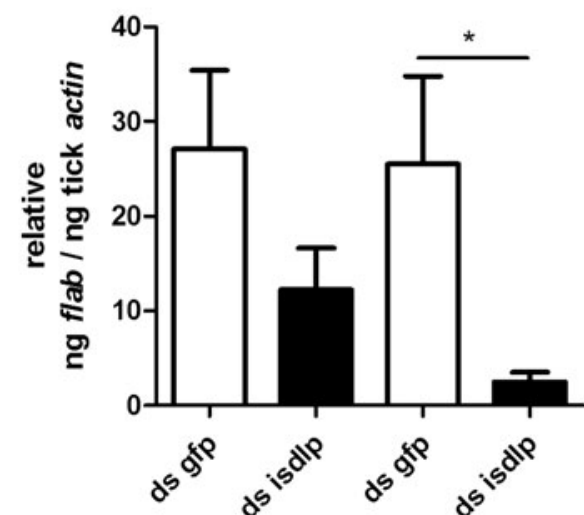

Fig. 4 Silencing of ISDLP expression by RNA interference results in decreased B. burgdorferi burden in the salivary glands and in murine skin. Double-stranded isdlp (ds $i s d l p$ ) or ds $g f p$ as a control was injected through the anal pore $3 \mathrm{~h}$ prior to B. burgdorferi-infected I. scapularis challenge (five ticks/mouse, five mice per experiment). Mice were sacrificed after 14 days. a RT-qPCR assessment of isdlp expression in the gut. b Engorgement weights of ticks post-feeding. Each data point represents one tick. A tick was considered female when $>3.5 \mathrm{mg}$. c
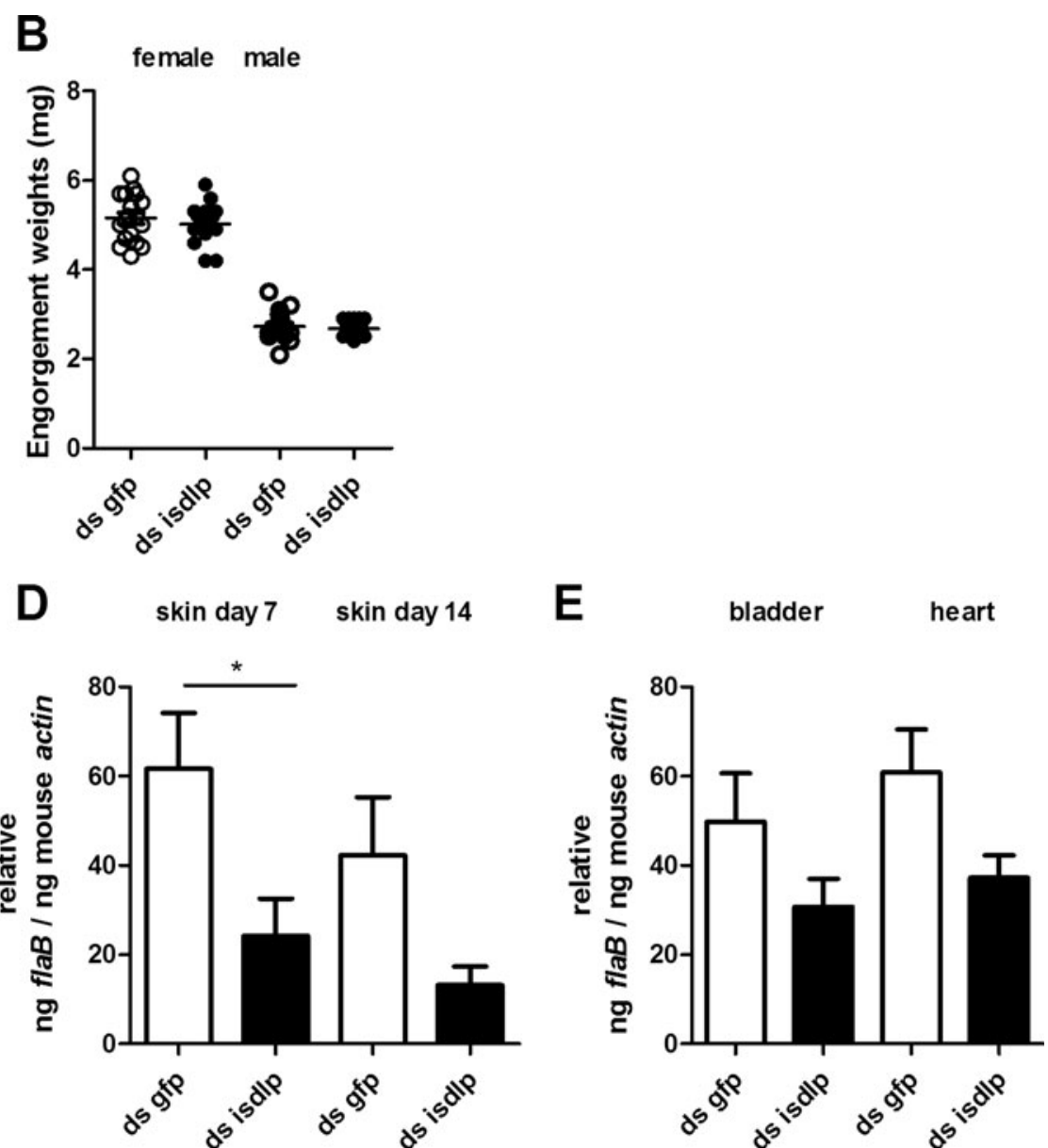

B. burgdorferi burden in tick guts and salivary glands post-feeding. d, e qPCR assessment of B. burgdorferi burden in the murine skin at 7 days and in the skin, bladder, and heart at 14 days post-tick feeding. The error bars represent mean \pm SEM, and the mean values that were significantly different in a two-tailed non-parametric Mann-Whitney test are indicated by an asterisk $(p \leq 0.05)$. The pooled results of two independent mouse experiments are shown

ISDLP did not impair $B$. burgdorferi migration to the salivary glands and did not reduce transmission to the murine host. There are several explanations for the discrepancy between our RNAi experiment and immunization experiment. While RNAi-mediated silencing is initiated prior to and during tick feeding, sufficient antibody uptake by the tick from the host might take more than 24-36 h coincident with the arrival of blood meal into the tick gut [22]. Thus, delayed entry of antibodies might allow B. burgdorferi to exploit the gut ISDLP and continue its migration from the gut. In order to prevent B. burgdorferi egress from the gut, anti-ISDLP antibodies have to significantly neutralize ISDLP's interaction with B. burgdorferi. Immunofluorescence microscopy suggests that ISDLP is ubiquitously represented on the tick gut. Affinity of the antibody binding as well as amounts of antibody that enter the gut would determine the successful 
abrogation of $B$. burgdorferi-ISDLP interaction. In addition, other possibilities that impaired a protective effect of antiISDLP antibodies could be the inaccessibility of the protective epitope within the tick or the inability of the generated antibodies to block the interaction between ISDLP and $B$. burgdorferi. The latter possibility is supported by in vitro observations that binding of ISDLP to $B$. burgdorferi could not be blocked by anti-ISDLP antibodies (Online Resource 2). More research to identify specific regions of ISDLP that interact with $B$. burgdorferi as well as the $B$. burgdorferi ligand that interacts with ISDLP would be informative for effectively blocking ISDLP-B. burgdorferi interaction through antibodies.

While several tick proteins with pharmacological functions critical for tick feeding and B. burgdorferi transmission have been identified [23], vaccine targeting has been confounded by the functional and structural paralogy of the tick transcriptome [24]. Recent efforts have increased our understanding of tick- $B$. burgdorferi interactions that facilitate $B$. burgdorferi migration within the tick. Vaccines targeting these $B$. burgdorferi-interacting tick proteins provide another avenue to interrupt $B$. burgdorferi transmission. It is becoming evident that $B$. burgdorferi exploits multiple tick proteins to temporally and spatially orchestrate its migration from the gut, entry into salivary glands, and transmission to the host. Elucidation of $B$. burgdorferi-interacting tick proteins that facilitate the various aspects of transmission would help design an optimal combination of vaccine targets that would provide a synergistic impairment of transmission to the vertebrate host.

Acknowledgments We sincerely thank Al Mennone at Yale University for his useful suggestions for microscopy. We are grateful to Kathleen DePonte and Jingyi Pan for their excellent technical assistance.

\section{Compliance with ethical standards}

Funding This work was supported by grant funded from the National Institute of Allergy and Infectious Diseases at the National Institutes of Health [AI076705 to S.N.], the Netherlands Organization for Health Research and Development (ZonMw) [91611065 to J.W.H.], and the European Union's Seventh Programme for research, technological development, and demonstration [602272, project ANTIDotE to J.W.H.]. E.F. is an investigator of the Howard Hughes Medical Institute.

Ethical statement The animals used in the described experiments were housed and handled under the Guide for the Care and Use of Laboratory Animals of the National Institutes of Health. The animal experimental protocol was approved by the Yale University's Institutional Animal Care and Use Committee (protocol number 2008-07941, approval date: 31 March 2014). In addition, the mice that were used for immunization experiments were housed and handled under the approval of the Animal Care and Use Committee of the University of Amsterdam (DIX103179). The manuscript does not contain clinical studies or patient data.

Conflict of interest The authors declare that they have no competing interests.
Open Access This article is distributed under the terms of the Creative Commons Attribution 4.0 International License (http:// creativecommons.org/licenses/by/4.0/), which permits unrestricted use, distribution, and reproduction in any medium, provided you give appropriate credit to the original author(s) and the source, provide a link to the Creative Commons license, and indicate if changes were made.

\section{References}

1. Steere AC, Coburn J, Glickstein L (2004) The emergence of Lyme disease. J Clin Invest 113(8):1093-1101

2. de la Fuente J, Estrada-Pena A, Venzal JM, Kocan KM, Sonenshine DE (2008) Overview: ticks as vectors of pathogens that cause disease in humans and animals. Front Biosci 13:6938-6946

3. Estrada-Pena A, Jongejan F (1999) Ticks feeding on humans: a review of records on human-biting Ixodoidea with special reference to pathogen transmission. Exp Appl Acarol 23(9):685-715

4. Pal U, Li X, Wang T, Montgomery RR, Ramamoorthi N, Desilva AM, Bao F, Yang X, Pypaert M, Pradhan D et al (2004) TROSPA, an Ixodes scapularis receptor for Borrelia burgdorferi. Cell 119(4): $457-468$

5. Radolf JD, Caimano MJ, Stevenson B, Hu LT (2012) Of ticks, mice and men: understanding the dual-host lifestyle of Lyme disease spirochaetes. Nat Rev Microbiol 10(2):87-99

6. Dunham-Ems SM, Caimano MJ, Pal U, Wolgemuth CW, Eggers CH, Balic A, Radolf JD (2009) Live imaging reveals a biphasic mode of dissemination of Borrelia burgdorferi within ticks. J Clin Invest 119(12):3652-3665

7. Plotkin SA (2011) Correcting a public health fiasco: the need for a new vaccine against Lyme disease. Clin Infect Dis 52(Suppl 3): s271-s275

8. Hovius JW, van Dam AP, Fikrig E (2007) Tick-host-pathogen interactions in Lyme borreliosis. Trends Parasitol 23(9):434-438

9. Schuijt TJ, Coumou J, Narasimhan S, Dai J, Deponte K, Wouters D, Brouwer M, Oei A, Roelofs JJ, van Dam AP et al (2011) A tick mannose-binding lectin inhibitor interferes with the vertebrate complement cascade to enhance transmission of the Lyme disease agent. Cell Host Microbe 10(2):136-146

10. Dai J, Wang P, Adusumilli S, Booth CJ, Narasimhan S, Anguita J, Fikrig E (2009) Antibodies against a tick protein, Salp15, protect mice from the Lyme disease agent. Cell Host Microbe 6(5):482492

11. Dai J, Narasimhan S, Zhang L, Liu L, Wang P, Fikrig E (2010) Tick histamine release factor is critical for Ixodes scapularis engorgement and transmission of the Lyme disease agent. PLoS Pathog 6(11), e1001205. doi:10.1371/journal.ppat.1001205

12. Narasimhan S, Coumou J, Schuijt TJ, Boder E, Hovius JW, Fikrig E (2014) A tick gut protein with fibronectin III domains aids Borrelia burgdorferi congregation to the gut during transmission. PLoS Pathog 10(8), e1004278. doi:10.1371/journal.ppat.1004278

13. Narasimhan S, Perez O, Mootien S, DePonte K, Koski RA, Fikrig E, Ledizet M (2013) Characterization of ixophilin, a thrombin inhibitor from the gut of Ixodes scapularis. PLoS One 8(7), e68012. doi:10.1371/journal.pone.0068012

14. Ibraghimov-Beskrovnaya O, Ervasti JM, Leveille CJ, Slaughter CA, Sernett SW, Campbell KP (1992) Primary structure of dystrophin-associated glycoproteins linking dystrophin to the extracellular matrix. Nature 355(6362):696-702

15. Schmit VL, Patton TG, Gilmore RD Jr (2011) Analysis of Borrelia burgdorferi surface proteins as determinants in establishing host cell interactions. Front Microbiol 2:141

16. Coburn J, Leong J, Chaconas G (2013) Illuminating the roles of the Borrelia burgdorferi adhesins. Trends Microbiol 21(8):372-379 
17. Constantin B (2014) Dystrophin complex functions as a scaffold for signalling proteins. Biochim Biophys Acta 1838(2):635-642

18. Spence HJ, Dhillon AS, James M, Winder SJ (2004) Dystroglycan, a scaffold for the ERK-MAP kinase cascade. EMBO Rep 5(5):484489

19. Rambukkana A, Salzer JL, Yurchenco PD, Tuomanen EI (1997) Neural targeting of Mycobacterium leprae mediated by the G domain of the laminin-alpha2 chain. Cell 88(6):811-821

20. Cao W, Henry MD, Borrow P, Yamada H, Elder JH, Ravkov EV, Nichol ST, Compans RW, Campbell KP, Oldstone MB (1998) Identification of alpha-dystroglycan as a receptor for lymphocytic choriomeningitis virus and Lassa fever virus. Science 282(5396): 2079-2081

21. Hallstrom T, Haupt K, Kraiczy P, Hortschansky P, Wallich R, Skerka C, Zipfel PF (2010) Complement regulator-acquiring surface protein 1 of Borrelia burgdorferi binds to human bone morphogenic protein 2, several extracellular matrix proteins, and plasminogen. J Infect Dis 202(3):490-498

22. Anderson JF, Magnarelli LA (2008) Biology of ticks. Infect Dis Clin N Am 22(2): 195-215

23. Hovius JW, Levi M, Fikrig E (2008) Salivating for knowledge: potential pharmacological agents in tick saliva. PLoS Med 5(2), e43. doi:10.1371/journal.pmed.0050043

24. Ribeiro JM, Alarcon-Chaidez F, Francischetti IM, Mans BJ, Mather TN, Valenzuela JG, Wikel SK (2006) An annotated catalog of salivary gland transcripts from Ixodes scapularis ticks. Insect Biochem Mol Biol 36(2):111-129

25. Schultz J, Milpetz F, Bork P, Ponting CP (1998) SMART, a simple modular architecture research tool: identification of signaling domains. Proc Natl Acad Sci 95(11):5857-5864

26. Kelley LA, Sternberg MJ (2009) Protein structure prediction on the Web: a case study using the Phyre server. Nat Protoc 4(3):363-371 